\title{
Recombinant receptor activator of nuclear factor $\kappa B$ exhibits more marked inhibitory effects on osteoclasts compared with recombinant osteoprotegerin in vitro and in vivo
}

\author{
QI XIONG ${ }^{1 *}$, LINGLI XIN $^{2 *}$, LICHENG ZHANG $^{1}$, PEIFU TANG $^{1}$ and LIHAI ZHANG ${ }^{1}$ \\ ${ }^{1}$ Department of Orthopedics, General Hospital of Chinese PLA, Beijing 100853; ${ }^{2}$ Department of Obstetrics and Gynecology, \\ The Second Artillery General Hospital of Chinese PLA, Beijing 100088, P.R. China
}

Received May 15, 2014; Accepted February 3, 2015

DOI: $10.3892 / \mathrm{mmr} .2015 .3407$

\begin{abstract}
The aim of the present study was to compare the osteoclast-inhibiting ability of recombinant osteoprotegerin (OPG) protein (rhOPG-Fc) and recombinant receptor activator of nuclear factor $\kappa \mathrm{B}$ (rhRANK) in vitro and in vivo. Osteoclasts were cultured with either rhOPG-Fc or rhRANK for 9 days. The number of tartrate-resistant acid phosphatase (TRAP)-positive multinucleated cells and resorption pits in bone slices were then counted. In the in vivo investigation, female mice were bilaterally ovariectomized (OVX) and intraperitoneally injected with $3 \mathrm{mg} / \mathrm{kg}$ rhOPG-Fc or rhRANK for 12 weeks, respectively. Bone metabolism, bone mineral density and microstructure changes were then evaluated. The number of TRAP-positive cells and bone resorption pits decreased significantly following culture with either rhOPG-Fc or rhRANK, and this was more marked following culture with rhRANK compared with rhOPG-Fc. The levels of calcium and alkaline phosphatase in the serum were similar pre-OVX and after 12 weeks of treatment, while the levels of phosphorus in the serum were higher following treatment with rhRANK compared with rhOPG. The bone mineral density (BMD) of the whole body, femoral neck and L4 lumbar vertebral body in the mice treated with either rhOPG-Fc or rhRANK increased markedly. In addition, the mice treated with rhRANK exhibited significantly higher BMD in the femoral neck and lumbar vertebral body compared with those treated with rhOPG-Fc. Microcomputed tomography analysis demonstrated that the mice treated with rhRANK exhibited an increased bone
\end{abstract}

Correspondence to: Dr Peifu Tang or Dr Lihai Zhang, Department of Orthopedics, General Hospital of Chinese PLA, 28 Fuxing Road, Haidian, Beijing 100853

E-mail: pftang301@126.com

E-mail: zhanglihai@gmail.com

${ }^{*}$ Contributed equally

Key words: osteoclast, osteoporosis, receptor activator of nuclear factor $\kappa \mathrm{B}$, osteoprotegerin volume and structure model index, and decreased trabecular spacing compared with those treated with rhOPG-Fc. rhRANK increased the inhibition of osteoclast differentiation and bone resorption, and rescued OVX-induced osteoporosis more effectively compared with rhOPG-Fc.

\section{Introduction}

The skeletal system is restructured at a high rate, with $\sim 10 \%$ of the total bone content being replaced each year in adult vertebrates (1). This process is called bone remodeling, which depends on a delicate balance between osteoblast bone formation and osteoclast bone resorption (2). An imbalance which favors osteoclast bone resorption leads to skeletal disorders, including osteoporosis and autoimmune arthritis $(3,4)$. Osteoporosis is a common bone disease, characterized by reduced bone density and altered trabecular microarchitecture, which leads to increased risk of fracture (5). As the increased risk of fracture has detrimental outcomes in terms of mortality rates and health care costs, several pharmaceutical antiresorptive measures have been developed and used clinically to suppress trabecular bone loss (6).

Osteoclasts, the only bone resorptive cell known to date, are multinucleated cells, which derive from hematopoietic cells of the monocyte/macrophage lineage (7). The unraveling of osteoprotegerin (OPG)/receptor activator of nuclear factor $\kappa \mathrm{B}(\mathrm{RANK}) / \mathrm{RANK}$ ligand (RANKL) system provides a better understanding of the mechanism undedryling osteoclast differentiation and function (8). RANKL, a member of the tumor necrosis factor (TNF) family, activates the receptor, RANK, which is present on osteoclasts and osteoclast precursors and promotes the proliferation, differentiation and maturation of osteoclasts, leading to bone resorption $(9,10)$. RANKL-knockout mice exhibit an osteopetrotic phenotype, which is characterized by the absence of osteoclasts (11). The degree of bone erosion in RANKL-knockout mice is significantly reduced compared with that observed in wild-type mice (12). OPG, a soluble decoy receptor from the TNF receptor family, binds RANKL and prevents the activation of the RANK receptor $(8,13)$. OPG-knockout mice exhibit marked bone loss accompanied by a lack of trabecular bone in their femurs due to enhanced osteoclastgenesis (14). 
RANKL inhibitors offer promise as therapeutic agents for the treatment of a wide range of bone loss disorders, particularly osteoporosis. Systemic RANKL blockade using OPG, OPG-Fc fusion protein, or inhibitory RANK antibodies have been successfully used to treat osteoprotic bone diseases. Wang et al demonstrated that recombinant OPG-Fc had a marked inhibitory effect on osteoclast differentiation, and ameliorated osteoporosis (15). Previous studies have also found that RANK exists in the peripheral blood in the form of soluble protein in addition to transmembrane protein $(16,17)$. When combined with RANKL, soluble RANK does not stimulate osteoclast differentiation or bone resorption (18). A study by Tang et al indicated that recombinant (rh)RANK inhibits osteoclast differentiation and prevents bone loss caused by ovariectomy (OVX) (19). Taken together, these findings revealed that rhRANK and rhOPG-Fc effectively bind to RANKL, inhibiting the activity of osteoclasts and rescuing osteoporosis. However, few reports have compared the abilities of rhRANK and rhOPG-Fc in inhibiting RANKL.

In our previous study, it was observed that rhRANK significantly inhibited RAW264.7 osteoclast precursor cells from differentiating into mature osteoclasts compared with rhOPG-Fc. The present study compared the capacities of rhRANK and rhOPG-Fc to inhibit osteoclasts and rescue OVX-induced osteoporosis.

\section{Materials and methods}

Osteoclast culture. The present study was approved by the General Hospital of Chinese PLA Ethical Committee (Beijing, China). The isolation of osteoclasts was performed, as previously described by Hefley et al (20). Briefly, the osteoclasts were mechanically separated from the long bones of newborn fetal mice. The bones were obtained and placed in cold Hank's buffer, following which the bones were dissected free of muscle and periosteum, the epiphyses were removed, and the diaphysis were sectioned with a scalpel in separating medium. The samples were centrifuged at $\sim 100 \mathrm{x} \mathrm{g}$ for $3 \mathrm{~min}$ at room temperature. Following the precipitation of large fragments to the bottom of the test tube, the cell suspension was removed, diluted with Dulbecco's modified Eagle's medium (DMEM) to $1 \times 10^{5} / \mathrm{ml}$, seeded into six-well plates with either coverslips or bone slices, and incubated at $37^{\circ} \mathrm{C}$ under $5 \% \mathrm{CO}_{2}$ in air. The culture medium contained DMEM with $10 \%$ fetal bovine serum (FBS), and was refreshed every 2 days. The six-well plates with coverslips or bone slice were divided into three groups: The control group, comprising cells cultured without rhRANK or rhOPG-Fc; the RANK group, comprising cells cultured with $10^{-5} \mathrm{~g} / 1 \mathrm{rhRANK}$; and the OPG group, comprising cells cultured with $10^{-5} \mathrm{~g} / 1 \mathrm{rhOPG}-\mathrm{Fc}$.

Bone slice preparation. The bovine femur (Walmart Supermarket, Beijing, China) was cut into $6 \mathrm{~mm} \times 6 \mathrm{~mm}$ diameter, $100 \mu \mathrm{m}$ thick slices using a Leica SP1600 (Leica Biosystems, Nussloch, Germany), and were then polished by ultrasonication (KQ-100B; Kunshan Ultrasonic Instruments Co., Ltd., Kunshan, China) with double distilled water three times for $30 \mathrm{~min}$, sterilized with ${ }^{60} \mathrm{Co}$ radiation (GWXJ80 ${ }^{60} \mathrm{Co}$ irradiator; General Hospital of Chinese PLA) and stored at $37^{\circ} \mathrm{C}$ until used.
Counting of mature osteoclasts and resorption pits. After 9 days, the cultured cells were subjected to tartrate-resistant acid phosphatase (TRAP) staining [Acid Phosphatase, Leukocyte (TRAP) kit; Sigma-Aldrich, St. Louis, MO, USA] at $37^{\circ} \mathrm{C}$ in an atmosphere containing $5 \% \mathrm{CO}_{2}$ to identify the formation of osteoclast-like cells. Toluidine blue (Huayueyang Biotechnology, Beijing, China) staining was used to identify bone resorption pits. The number of TRAP-positive cells and resorption pits in the bone slices were counted.

The TRAP staining was performed using a TRAP staining kit, according to the manufacturer's instructions. The morphologies of the osteoclasts were observed under an inverted phase contrast microscope (IX70; Olympus, Tokyo, Japan). The multinucleated giant cells containing more than two positively stained nuclei were termed mature osteoclasts. A total of 10 randomly selected visual fields per group were visualized under a light microscope (IX70; magnification, x100) and the average number of osteoclasts was recorded in each group.

The bone slices in the culture system were fixed with $2.5 \%$ glutaraldehyde (Sigma-Aldrich) and stained with toluidine blue. The positively stained lacunae in the entire bone slices were counted under a light microscope (magnification, $\mathrm{x} 100$ ). The average number of resorption pits was recorded in each group.

Experimental animals. Female C57 mice (6-week-old; 20-30 g) provided by the Experimental Animal Center, Chinese General Hospital of PLA (Beijing, China), were used in the present study. The mice were maintained at room temperature, were subjected to a $12 \mathrm{~h}$ light/12 h dark cycle and were fed ad libitum. The mice were randomly divided into three groups ( $n=10 /$ group). In the OVX group, the mice received bilateral ovariectomies and intraperitoneal injection of $1.0 \mathrm{ml}$ vehicle buffer (phosphate-buffered saline) three times weekly for 12 weeks; in the RANK group, the mice received bilateral OVX and intraperitoneal injection of $1.0 \mathrm{ml}$ rhRANK ( $5 \mathrm{mg} / \mathrm{kg}$ body weight) three times weekly for 12 weeks; in the OPG group, the mice received bilateral OVX and intraperitoneal injection of $1.0 \mathrm{ml} \mathrm{rhOPG}-\mathrm{Fc}(5 \mathrm{mg} / \mathrm{kg}$ body weight) three times weekly for 12 weeks. The mice were sacrificed by cervical dislocation. All animal experiments were performed according to the animal care guidelines, Department of Health, China, with the approval of the PLA General Hospital Ethical Committee on Animal Research.

OVX surgery. The ovariectomized animal models were established as follows. General anesthesia was administered to female mice through intraperitoneal injection of $3 \%$ sodium pentobarbital (1 ml/kg; Merck, Darmstadt, Germany). Subsequently, the mice were fixed in a prone position and OVX surgery was performed. Briefly, an incision was made at the middle of the back, and the uterus $(2-3 \mathrm{~mm}$ in diameter) and ovary were exposed. The ovary was carefully separated and resected, and the fascia was sutured. The other ovary was resected in the same way. Following surgery, 80,000 units penicillin was injected intramuscularly daily for 3 days to prevent infection. For the sham group, the uterine tubes were ligated and the ovaries were separated, however, no OVX was performed. At 1 week following surgery, the 
mice were administered with either the rhRANK, rhOPG or the vehicle buffer. All the animals were reared under the same conditions and were provided standard feed, containing $1.66 \%$ calcium $(\mathrm{Ca})$ and $1.24 \%$ phosphorus $(\mathrm{P})$.

Serum biochemical assays. Anesthesia (3\% sodium pentobarbital; $1 \mathrm{ml} / \mathrm{kg}$ ) was administered to each mouse by intraperitoneal injection. Subsequently, $0.5 \mathrm{ml}$ blood was collected from the tail vein of each mouse using a plastic heparinized syringe. The blood was centrifuged at 3,000 xg for $15 \mathrm{~min}$ at room temperature. The serum was obtained and filtered through a $0.22 \mu \mathrm{m}$ filter (Corning, Inc., Corning, NY, USA) for sterilization and to removing fragments of platelets. Blood was collected at day 0 and 12 weeks after treatment. The levels of serum alkaline phosphatase (ALP), calcium and phosphorus were evaluated. The levels of calcium and phosphorus were measured by standard laboratory assessments. The level of ALP was measured by ELISA kit (Mouse ALP ELISA kit; Abbexa Ltd., Cambridge, UK) according to the manufacturer's instructions.

Bone mineral density measurements. At 12 weeks after treatment, bone mineral density (BMD) measurements were performed using a Norland XR-36 bone density instrument (Norland Medical Systems, Fort Atkinson, WI, USA) and scanned using the small animal model function. The BMD of the whole body, femoral neck and L4 vertebral bodies of each mouse were measured.

Micro-computed tomography (micro-CT) measurements. The trabeculae of the lumbar vertebrate and distal femora were analyzed by micro-CT (Micro-CT 40; Scanco Medical, Brüttisellen, Switerland). The energy and intensities were to $40 \mathrm{kVp}$ and $250 \mu \mathrm{A}$, respectively. Following fixation in $70 \%$ alcohol, the microstructures of the lumbar vertebral body and femur were scanned. Three-dimensional trabecular analysis was performed and the percentage of trabecular bone volume (BV/TV\%), trabecular thickness (Tb.Th), trabecular number (Tb.N), trabecular spacing (Tb.Sp), and structure model index (SMI) were analyzed using Scanco version 5.0 software (Scanco Medical, Brüttisellen, Switzerland).

Statistics analysis. All data were analyzed using SPSS 17.0 software (SPSS, Inc. Chicago, IL, USA). All results are expressed as the mean \pm standard error of the mean. Analysis of variance and Fisher's protected least significant difference test were used in multi-group comparisons. $\mathrm{P}<0.05$ was considered to indicate a statistically significant difference.

\section{Results}

Effect of rhOPG-Fc and rhRANK on osteoclasts. The results of TRAP-positive cell counting revealed significant differences among the three groups $(\mathrm{F}=32.9 ; \mathrm{P}<0.05)$. The numbers of TRAP-positive cells in the RANK group and OPG group were significantly lower compared with the control group, and the number of TRAP-positive cells in the RANK group was significantly less compared with the OPG group. (Table I; Fig. 1A)

The results of the bone resorption pit counting revealed consistent results. The number of resorptive pits was signifi-
A

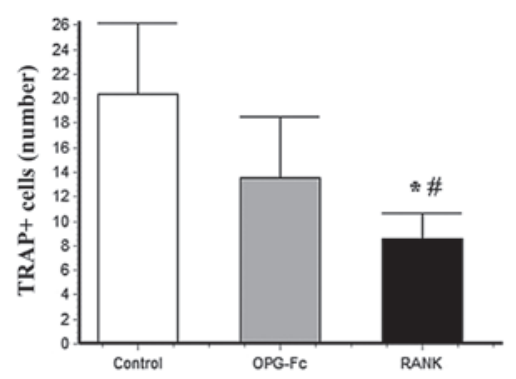

$\mathbf{B}$

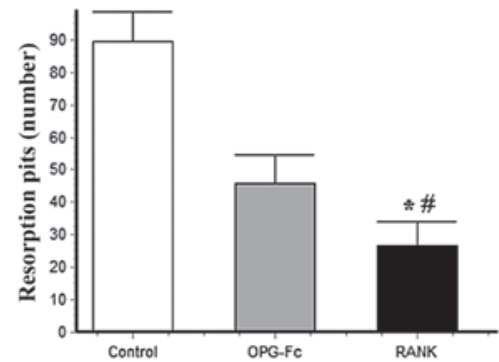

Figure 1. Following culture for 9 days, TRAP-positive cells and bone resorption pits were counted. The RANK group and OPG group had significantly fewer TRAP-positive cells and bone resorption pits compared with the control group. The RANK group had significantly fewer TRAP-positive cells and bone resorption pits compared with the OPG group. (A) Number of TRAP-positive cells 9 days after culture. (B) Number of resorption pits on the bone slices. Data are expressed as the mean \pm standard error of the mean of each group. ${ }^{*} \mathrm{P}<0.05$, compared with the control and OPG-Fc groups. ${ }^{\text {"}} \mathrm{P}<0.05$, compared with the OPG group. RANK, receptor activator of nuclear factor $\mathrm{\kappa B}$; OPG, osteoprotegerin; TRAP, tartrate-resistant acid phosphatase.

cantly different among the three groups $(\mathrm{F}=143.0 ; \mathrm{P}<0.05)$. The numbers of bone resorption pits in the RANK group and OPG group were significantly lower compared with the control group. In addition, the number of bone resorption pits in the RANK group was significantly less compared with that observed in the OPG group.

Changes in the levels of calcium, phosphorus and ALP in the serum. The levels of calcium and ALP in serum were similar in all three groups prior to and following OVX (Fig. 2). The results also demonstrated no significantly changes 12 weeks after surgery in any of the groups.

No significant differences were observed in the serum levels of phosphorus among the groups pre-OVX $(\mathrm{F}=1.19 ; \mathrm{P}=0.32$; Table II), however, significant differences were observed among the groups 12 weeks after $\mathrm{OVX}(\mathrm{F}=5.44 ; \mathrm{P}=0.01)$, with the lowest level of phosphorus in the OPG group (Fig. 2). When comparing the levels of phosphorus pre-OVX and post-OVX in each group, the results revealed that there were significant decreases in the OPG $(\mathrm{t}=3.45 ; \mathrm{P}=0.003)$ and RANK $(\mathrm{t}=2.39$; $\mathrm{P}=0.03$ ) groups, while no change was observed in the OVX group.

BMD measurements. The BMD of the whole body, femur and lumbar vertebral body of each mouse prior to OVX and following OVX were measured. There were no significant differences in the whole body BMD between the groups pre-OVX. At 12 weeks after OVX, the values of whole body $\mathrm{BMD}$ in each group were significantly different $(\mathrm{F}=52.37$; 
Table I. Numbers of multinuleated TRAP-positive cells and bone resorption pits.

\begin{tabular}{llrr}
\hline Characteristic & Control (n) & OPG (n) & RANK (n) \\
\hline TRAP-positive & $20.4 \pm 5.75$ & $13.5 \pm 4.94$ & $8.6 \pm 2.06^{\mathrm{ab}}$ \\
Resorption pits & $89.5 \pm 9.15$ & $45.9 \pm 8.79$ & $26.7 \pm 7.51^{\mathrm{ab}}$
\end{tabular}

Data are expressed as the mean \pm standard error of the mean $(\mathrm{n}=10)$. ${ }^{\mathrm{a}} \mathrm{P}<0.05$, compared with the control and $\mathrm{OPG}$ groups; ${ }^{\mathrm{b}} \mathrm{P}<0.05$ compared with the OPG group. TRAP, tartrate-resistant acid phosphatase; OPG, osteoprotegerin; RANK, receptor activator of nuclear factor $\kappa \mathrm{B}$.

Table II. Biochemical markers in serum pre-OVX and 12 weeks post-OVX (mean \pm standard deviation).

\begin{tabular}{|c|c|c|c|c|c|c|}
\hline \multirow[b]{2}{*}{ Group } & \multicolumn{2}{|c|}{ Calcium (mmol/l) } & \multicolumn{2}{|c|}{ Phosphorus (mmol/l) } & \multicolumn{2}{|c|}{$\operatorname{ALP}(\mathrm{U} / \mathrm{l})$} \\
\hline & Pre-OVX & Post-OVX & Pre-OVX & Post-OVX & Pre-OVX & Post-OVX \\
\hline OVX & $2.41 \pm 0.09$ & $2.35 \pm 0.17$ & $3.1 \pm 0.54$ & $2.9 \pm 0.24$ & $89.9 \pm 23.5$ & $83.8 \pm 22.9$ \\
\hline OPG & $2.42 \pm 0.11$ & $2.34 \pm 0.11$ & $3.3 \pm 0.48^{\mathrm{a}}$ & $2.7 \pm 0.27^{\mathrm{c}}$ & $79.2 \pm 19.9$ & $88.2 \pm 24.3$ \\
\hline RANK & $2.37 \pm 0.08$ & $2.32 \pm 0.08$ & $3.4 \pm 0.26^{\mathrm{a}}$ & $3.1 \pm 0.30^{\mathrm{b}}$ & $80.7 \pm 21.1$ & $85.5 \pm 25.2$ \\
\hline
\end{tabular}

Data are expressed as the mean \pm standard error of the mean. ${ }^{a} \mathrm{P}<0.05$, pre-OVX, vs. post-OVX in the same group; ${ }^{\mathrm{b}} \mathrm{P}<0.05$, RANK, vs. OVX and OPG groups; ${ }^{\mathrm{C}} \mathrm{P}<0.05, \mathrm{OPG}$, vs. RANK group. OVX, ovariectomized; ALP, alkaline phosphatase; OPG, osteoprotegerin; RANK, receptor activator of nuclear factor $\kappa \mathrm{B}$.

Table III. Bone mineral density of the whole body, femoral neck and L4 vertebral body.

\begin{tabular}{|c|c|c|c|c|c|c|}
\hline \multirow[b]{2}{*}{ Group } & \multicolumn{2}{|c|}{ Whole body $\left(\mathrm{g} / \mathrm{cm}^{2}\right)$} & \multicolumn{2}{|c|}{ Femoral neck $\left(\mathrm{g} / \mathrm{cm}^{2}\right)$} & \multicolumn{2}{|c|}{ L4 vertebral body $\left(\mathrm{g} / \mathrm{cm}^{2}\right)$} \\
\hline & Pre-OVX & Post-OVX & Pre-OVX & Post-OVX & Pre-OVX & Post-OVX \\
\hline OVX & $0.091 \pm 0.003^{\mathrm{a}}$ & $0.076 \pm 0.005$ & $0.092 \pm 0.004^{\mathrm{a}}$ & $0.081 \pm 0.004$ & $0.089 \pm 0.006^{\mathrm{a}}$ & $0.079 \pm 0.004$ \\
\hline OPG & $0.087 \pm 0.006$ & $0.089 \pm 0.002$ & $0.085 \pm 0.005^{\mathrm{a}}$ & $0.091 \pm 0.003^{c}$ & $0.085 \pm 0.005$ & $0.087 \pm 0.003^{\mathrm{c}}$ \\
\hline RANK & $0.089 \pm 0.005$ & $0.091 \pm 0.003^{\mathrm{b}}$ & $0.089 \pm 0.003$ & $0.094 \pm 0.008^{\mathrm{b}}$ & $0.086 \pm 0.009$ & $0.092 \pm 0.006^{\mathrm{b}}$ \\
\hline
\end{tabular}

Data are expressed as the mean \pm standard error of the mean. ${ }^{a} \mathrm{P}<0.05$ pre-OVX, vs. post-OVX in the same group; ${ }^{\mathrm{b}} \mathrm{P}<0.05 \mathrm{RANK}$, vs. OVX and OPG groups; ${ }^{\mathrm{P}}<0.05 \mathrm{OPG}$, vs. RANK group. OVX, ovariectomized; OPG, osteoprotegerin; RANK, receptor activator of nuclear factor $\kappa \mathrm{B}$.

$\mathrm{P}<0.05)$. The mice in the OVX group had the lowest BMD, and the BMD of the mice in the RANK and OPG groups were higher, however, no significant difference was observed between the RANK and OPG groups. When the preoperative $\mathrm{BMD}$ values were compared with the postoperative BMD values, the whole body BMD of the OVX group was significantly decreased $(\mathrm{t}=8.14 ; \mathrm{P}<0.001)$, however, no significant change was observed in the OPG group or the RANK group. (Table III; Fig. 3A and B).

The BMD of the femoral neck was also measured. The BMD values of the femoral neck in each group were similar pre-OVX. However, the BMD values among the groups were significantly different 12 weeks post-OVX and drug administration $(\mathrm{F}=20.35 ; \mathrm{P}<0.001)$. The femoral neck $\mathrm{BMD}$ values of mice in the RANK and OPG groups were significantly higher compared with the OVX group, with the mice in the RANK group exhibiting the highest femoral neck BMD. When compared with the OPG group, the RANK group had a significantly higher BMD value. The results also revealed that the femoral neck BMD values of mice in the OVX group decreased significantly $(\mathrm{t}=6.15 ; \mathrm{P}<0.001)$ between the preoperative and 12-week post-treatment period, and the femoral neck BMD of mice in the OPG group increased significantly $(\mathrm{t}=3.254 ; \mathrm{P}=0.004)$. No significant difference was observed in the RANK group. (Table III; Fig. 3C and D)

The BMD values of the L4 lumbar vertebral body in all three groups was similar prior to OVX. After 12 weeks treatment, there was a signficant difference between the BMD of the lumbar vertebral body in the OVX group compared with the other two groups $(\mathrm{F}=21.1 ; \mathrm{P}<0.001)$, with the RANK and OPG groups exhibiting higher vertebral body BMD values compared with the OVX group. When comparison was made between the preoperative and postoperative vertebral body BMD values, there was a significant reduction in the BMD of the mice in the OVX group, whereas the BMD of mice in the OPG $(\mathrm{t}=1.085, \mathrm{P}=0.2924)$ and $\mathrm{RANK}(\mathrm{t}=1.754$, $\mathrm{P}=0.0964)$ groups increased, although not significantly. (Table III; Fig. 3E and F) 
Table IV. Micro-computed tomography analysis of the distal femur.

\begin{tabular}{lccr}
\hline Group & OVX & OPG & RANK \\
\hline Trabecular thickness (mm) & $0.08 \pm 0.02$ & $0.10 \pm 0.08$ & $0.12 \pm 0.03$ \\
Trabecular spacing (mm) & $0.36 \pm 0.14$ & $0.24 \pm 0.12$ & $0.16 \pm 0.11$ \\
Bone volume (\%) & $22.1 \pm 3.54$ & $28.2 \pm 2.21$ & $37.5 \pm 3.95$ \\
Structure model index & $1.35 \pm 0.39$ & $0.82 \pm 0.48$ & $0.42 \pm 0.36$
\end{tabular}

Data are expressed as the mean \pm standard deviation. OVX, ovariectomized; OPG, osteoprotegerin; RANK, receptor activator of nuclear factor $\kappa \mathrm{B}$.

Table V. Micro-computed tomography analysis of the L4 vertebral body.

\begin{tabular}{lccr}
\hline Group & OVX & OPG & RANK \\
\hline Trabecular thickness (mm) & $0.10 \pm 0.01$ & $0.12 \pm 0.08$ & $0.14 \pm 0.15$ \\
Trabecular spacing (mm) & $0.37 \pm 0.12$ & $0.23 \pm 0.14$ & $0.12 \pm 0.12$ \\
Bone volume (\%) & $28.1 \pm 2.64$ & $34.2 \pm 4.15$ & $39.8 \pm 3.91$ \\
Structure model index & $1.23 \pm 0.31$ & $0.79 \pm 0.48$ & $0.45 \pm 0.25$ \\
\hline
\end{tabular}

Data are expressed as the mean \pm standard deviation. OVX, ovariectomized; OPG, osteoprotegerin; RANK, receptor activator of nuclear factor $\kappa \mathrm{B}$.
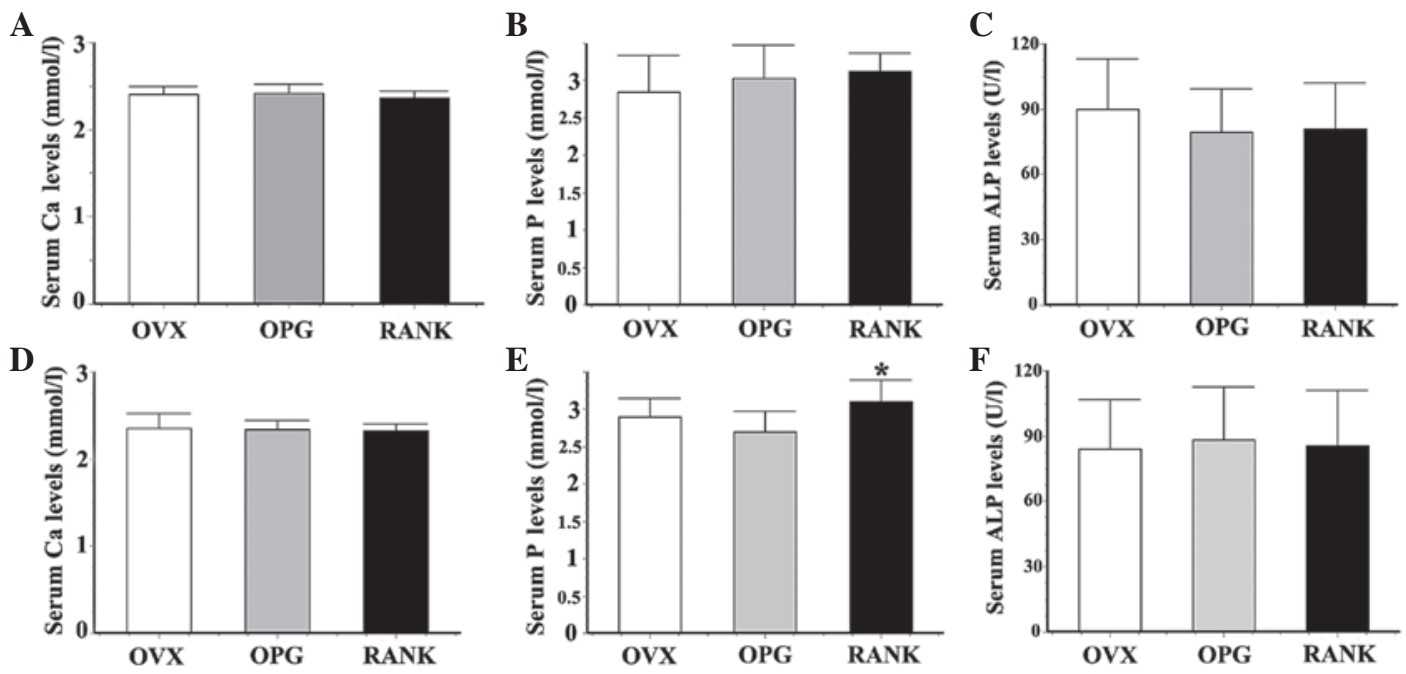

Figure 2. Serum biomarkers pre-OVX and 12 weeks post-drug administration. (A) Ca levels in the serum pre-OVX. (B) P levels in the serum pre-OVX. (C) ALP levels in the serum pre-OVX. (D) Ca levels in the serum 12 weeks after OVX. (E) P levels in the serum 12 weeks after OVX. (F) ALP levels in the serum 12 weeks after OVX. Data are expressed as the mean \pm standard error of the mean of each group. ${ }^{*} \mathrm{P}<0.05$ RANK, vs. OVX and OPG groups. OVX, ovariectomized; OPG, osteoprotegerin; RANK, receptor activator of nuclear factor $\mathrm{\kappa B}$. Ca, calcium; P, phosphorus; ALP, alkaline phosphatase.

Micro-CT Analysis of the lumbar vertebral body and femur. Quantification of the trabecular structures revealed that the mice in the OVX group exhibited significant osteoporosis, severely damaged trabecular structure, significantly narrowed Tb.Th, widened Tb.Sp and significantly reduced BV/TV\%. There were no statistically significantly differences in Tb.Th among the groups, however, the BV/TV\% was significantly higher in the OPG and RANK groups compared with the OVX group, and the BV/TV\% in the RANK group was significantly higher compared with the OPG group. The Tb.Sp and SMI values were statistically different among the groups, with the RANK group exhibiting the lowest values, and the OVX group exhibiting the highest values. However the Tb.Sp and SMI values in the RANK and OPG groups were significantly lower compared with the OVX group. (Table IV; Fig. 4A-D)

Micro-CT analysis of the lumbar vertebrae revealed that the trabecular structure was severely damaged, and the BV/TV\% and Tb.Th were decreased in the OVX group, while the Tb.Sp was widened. Intergroup comparison of the Tb.Sp and Tb.Th values demonstrated statistically significant differences among the groups, as the Tb.Sp values were significantly reduced and the Tb.Th values were significantly increased in the RANK 
A

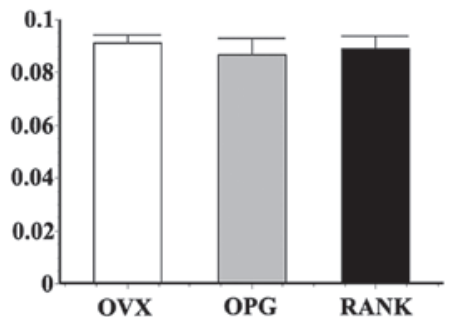

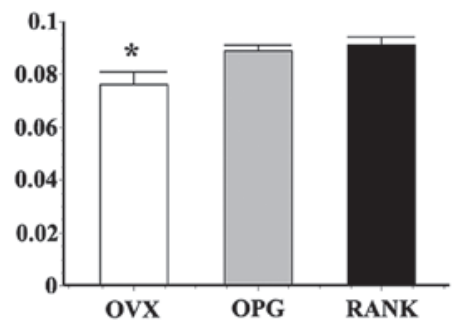

C
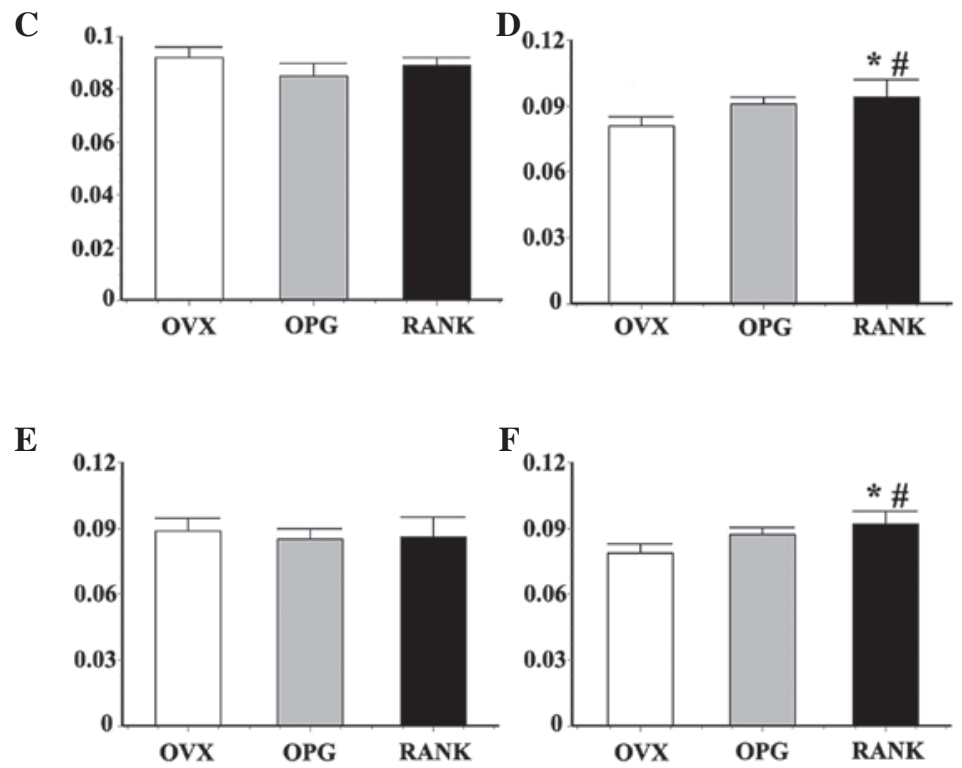

Figure 3. BMD of all the groups pre-OVX and 12 weeks after drug administration. (A) BMD of the whole body pre-OVX. (B) BMD of the whole body 12 weeks after drug administration. (C) BMD of the femoral neck pre-OVX. (D) BMD of the femoral neck 12 weeks after drug administration. (E) BMD of the L4 vertebral body pre-OVX. (F) BMD of the L4 vertebral body 12 weeks after drug administration. Data are expressed as the mean \pm standard error of the mean of each group. "P $<0.05$, compared with the OVX and OPG groups; ${ }^{*} \mathrm{P}<0.05$, RANK, vs. OPG group.
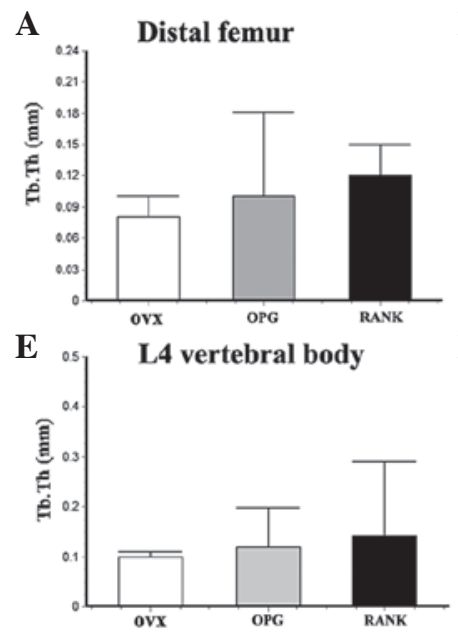
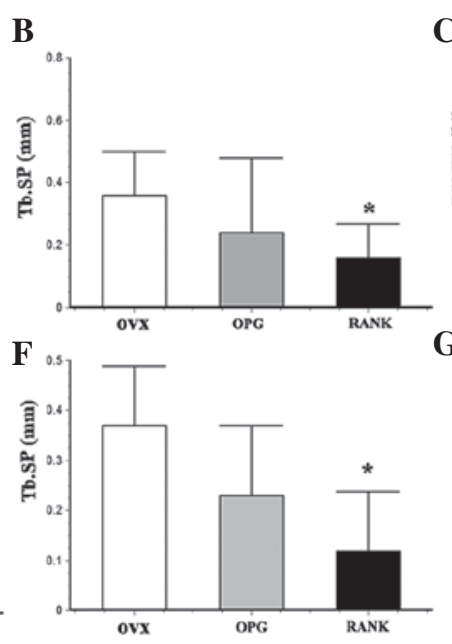

C
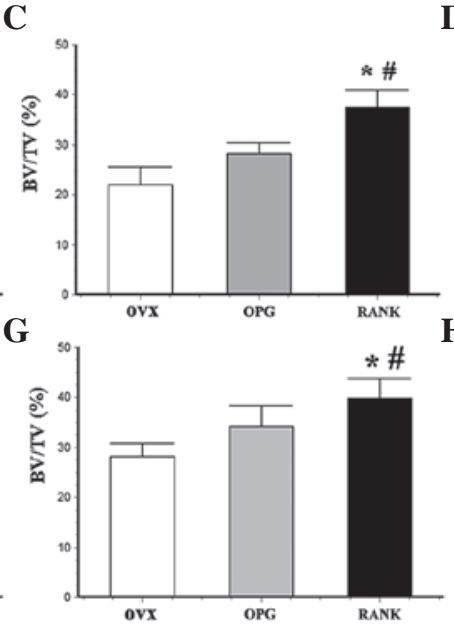

D

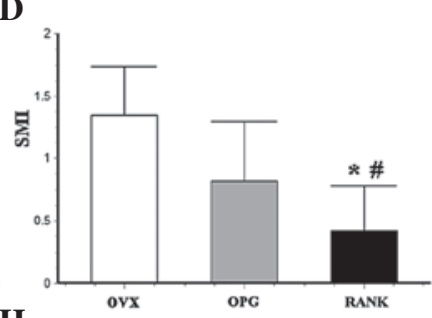

$\mathbf{H}$

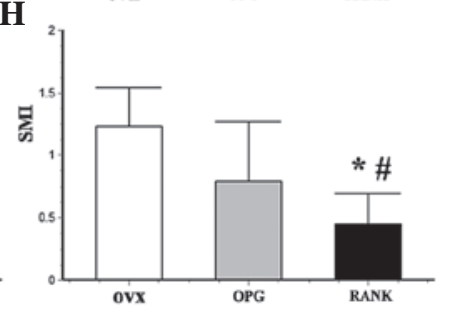

Figure 4. Micro-computed tomography analysis of the trabecular structures of all groups. (A-D) Bone parameters of the distal femur. (E-H) Bone parameters of the L4 vertebral body. Data are expressed as the mean \pm standard error of the mean of each group *P<0.05, compared with the OVX and OPG groups; ${ }^{\#} \mathrm{P}<0.05$ RANK, vs. OPG group. OVX, ovariectomized; OPG, osteoprotegerin; RANK, receptor activator of nuclear factor $\kappa B$. Tb.Th, trabecular thickness; Tb.Sp, trabecular spacing; SMI, structure model index.

and OPG groups compared with the OVX group. The results also revealed that the BV/TV\% in the RANK and OPG groups were significantly increased compared with the OVX group, and the RANK group exhibited a significantly higher BV/TV\% compared with the OPG group. The SMI in the RANK group was significantly lower compared with the OVX and OPG groups, and the SMI in the OPG group was significantly lower compared with the OVX group. (Table V; Fig. 4E-H) 


\section{Discussion}

rhRANK and rhOPG can inhibit osteoclast differentiation and bone resorption ex vivo and in vivo. In the present study, the inhibitory effect of these two agents on the differentiation of osteoclasts was compared. The results suggested that rhRANK has an increased ability, compared with rhOPG, in inhibiting osteoclasts and rescuing osteoporosis. In the present study, osteoclasts were isolated from the long bone of newborn fetal mice and were cultured with either rhRANK or rhOPG-Fc. Significantly less mature osteoclasts and fewer bone resorption pits were observed following treatment with rhRANK, compared with rhOPG. These findings on osteoclast inhibition by rhOPG and rhRANK are concordant with previous reports $(13,17)$. Previous studies have demonstrated that OPG competes with RANK in binding to RANKL. However, OPG can also bind to the tumor necrosis factor-related apoptosis-inducing ligand, while RANK exclusively binds to RANKL (21). As the molecular weights of rhRANK and rhOPG differ, the number of rhRANK molecules differs to that of rhOPG at the same mass concentration and it has also been reported that the affinity of RANK for RANKL is almost 200 times higher than OPG $(22,23)$. These factors may, in part, explain the results of the present study that RANK inhibited the activity of osteoclasts more effectively compared with rhOPG. Further detailed analysis is required to fully elucidate this observation.

As OVX-induced bone loss in mice and postmenopausal bone loss share several similar characteristics, the ovariectomized female mouse is the currently recognized animal model for osteoporosis (24). In order to compare the efficiency of the two anti-osteoporotic agents in vivo, the present study performed experiments on OVX mice. The results demonstrated that, 12 weeks after OVX, the calcium levels in the serum were not significantly different among the groups, which was in agreement with previous studies. Tang et al found that, following 12 weeks treatment, serum calcium levels were similar in mice treated with or without recombinant murine RANK (19). Wang et al also demonstrated no significant change between the urinary excretion of calcium (15). Taken together, these findings suggested that RANK and OPG did not affect the bone metabolism of calcium. Similarly, although the level of ALP was lower in the OVX group, no significant differences were observed in the ALP levels between the groups. ALP is a commonly used indicator of bone turnover and bone formation (25). The results demonstrated that bone formation in the OVX group was reduced, and that rhRANK and rhOPG-Fc ameliorated this reduction. However, phosphorus levels decreased significantly following treatment with either rhOPG or rhRANK, and were significantly different among the groups 12 weeks post OVX. These data regarding serum biomarkers suggested that the effects of rhRANK and rhOPG on bone metabolism were not significantly different.

Osteoporosis is characterized by low bone mass and the microarchitectural deterioration of bone tissue, particularly in cancellous bone $(26,27)$. The present study compared the BMD and changes in bone structure of OVX mice treated with the two agents. After 12 weeks treatment, the whole body BMD values were significantly different among the groups, with the whole body BMD of the OVX mice, treated with neither agent, significantly reduced, and those of the rhRANK- and OPGrh-treated mice notably increased following OVX. The results also demonstrated that rhRANK and rhOPG enhanced the whole body BMD to a similar degree. The BMD of the femoral neck in each group was then compared, which revealed that rhRANK and rhOPG rescued the reduction in BMD induced by OVX. The mice treated with RANK had significantly higher BMD values compared with those treated with rhOPG. In addition, the BMD of the lumbar vertebral body increased significantly following treatment with the two agents, and the mice treated with RANK had significantly higher lumbar vertebral body BMD values compared with those treated with OPG. However, no significant difference was observed between the BMD of the lumbar vertebrae prior to nor following treatment with the agents. All these results demonstrated that rhRANK and rhOPG increased the BMD of OVX mice and ameliorated osteoporosis-induced bone loss. In addition, rhRANK exhibited an increased capacity to rescue reduced bone loss compared with rhOPG. To investigate the efficiency of the two agents in rescuing microarchitectural damage, micro-CT analyses of the lumbar vertebral body and distal femora were performed. The results demonstrated that the OVX mice had severely damaged trabecular structure, narrowed Tb.Th, widened Tb.Sp and significantly reduced $\mathrm{BV} / \mathrm{TV} \%$ in the lumbar vertebral body and in distal femur, which are common bone architecture changes in osteoporosis. The changes in SMI, were also calculated, the results of which indicated that the cancellous bone structure of OVX mice exhibited a rod-like trabecular structure. The findings demonstrated that the OVX mice had serious osteoporosis. The mice treated with rhRANK and rhOPG-Fc exhibited normal cancellous structure, which was a plate-like trabecular structure. Quantitative analysis revealed that mice treated with rhRANK and rhOPG-Fc had significantly increased BV/TV\% and reduced Tb.Sp. The SMI values were also statistically diversified among the groups, with the rhRANK and rhOPG groups exhibiting significantly less SMI compared with the OVX group. The Tb.Sp and SMI values of the mice in the RANK group were significantly lower compared with those in the OPG group. The results of the present study demonstrated that rhRANK and rhOPG prevented the microarchitectural deterioration of bone, induced by OVX, which supported the possible use of these agent to treat osteoporosis. The mice treated with rhRANK exhibited higher BV and a sophisticated trabecular structure, which indicated that rhRANK had a more marked ability at inhibiting the resorption of bone by osteoclasts and in ameliorating osteoporosis.

In conclusion, the present study indicated that rhRANK and rhOPG are effective antagonists of RANKL, the results of the ex vivo experiments demonstrated that they inhibited osteoclast differentiation and bone resorption, with rhRANK exhibiting a more marked inhibitory effect compared with rhOPG. The in vivo experiments revealed that rhRANK and rhOPG rescued OVX-induced osteoporosis, of which rhRANK had a greater ability at ameliorating bone loss and microarchitectural deterioration. Therefore, rhRANK may be a more effective antiresorptive drug compared with rhOPG. 


\section{Acknowledgements}

Dr Qi Xiong, Dr Licheng Zhang, Professor Peifu Tang and Dr Lihai Zhang are supported by the National Natural Science Foundation of China (grant no. 31370947).

\section{References}

1. Manolagas SC: Birth and death of bone cells: Basic regulatory mechanisms and implications for the pathogenesis and treatment of osteroporosis. Endocr Rev 21: 115-137, 2000.

2. Takayanagi H: Osteoimmunology: shared mechanisms and crosstalk between the immune and bone systems. Nat Rev Immunol 7: 292-304, 2007.

3. Deal C: Bone loss in rheumatoid arthritis: systemic, periarticular, and focal. Curr Rheumatol Rep 14: 231-237, 2012.

4. Geusens P and Lems WF: Osteoimmunology and osteoporosis. Arthritis Res Ther 13: 242, 2011.

5. Yasuda H: RANKL, a necessary chance for clinical application to osteoporosis and cancer-related bone diseases. World J Orthod 4 207-217, 2013

6. Kawai M, Mödder UI, Khosla S and Rosen CJ: Emerging therapeutic opportunities for skeletal restoration. Nat Rev Drug Discov 10: 141-156, 2011

7. Mizuno A, Amizuka N, Irie K, et al: Severe osteoporosis in mice lacking osteoclastogenesis inhibitory factor/osteoprotegerin. Biochem Biophys Res Commun 247: 610-615, 1998.

8. Khosla S: Minireview: the OPG/RANKL/RANK system. Endocrinology 142: 5050-5055, 2001

9. Weitzmann MN: The Role of Inflammatory Cytokines, the RANKL/OPG Axis, and the Immunoskeletal Interface in Physiological Bone Turnover and Osteoporosis. Scientifica-Cairo 2013: 125705, 2013

10. Lacey DL, Timms E, Tan HL, et al: Osteoprotegerin ligand is a cytokine that regulates osteoclast differentiation and activation. Cell 93: 165-176, 1998.

11. Kong YY, Yoshida H, Sarosi I, et al: OPGL is a key regulator of osteoclastogenesis, lymphocyte development and lymph-node organogenesis. Nature 397: 315-323, 1999.

12. Pettit AR, Ji H, von Stechow D, et al: TRANCE/RANKL knockout mice are protected from bone erosion in a serum transfer model of arthritis. Am J Pathol 159: 1689-1699, 2001.

13. Shiotani A, Takami M, Itoh K, Shibasaki Y and Sasaki T: Regulation of osteoclast differentiation and function by receptor activator of NFkB ligand and osteoprotegerin. Anat Rec 268: 137-146, 2002.
14. Liu J, Xu K, Wen G, et al: Comparison of the effects of genistein and zoledronic acid on the bone loss in OPG-deficient mice. Bone 42: 950-959, 2008

15. Wang Y, Huang P, Tang PF, Chan KM and Li G: Alendronate (ALN) combined with osteoprotegerin (OPG) significantly improves mechanical properties of long bone than the single use of ALN or OPG in the ovariectomized rats. J Orthop Surg 6: 34, 2011

16. Zhang J, Dai J, Yao Z, Lu Y, Dougall W and Keller ET: Soluble receptor activator of nuclear factor kappaB $\mathrm{Fc}$ diminishes prostate cancer progression in bone. Cancer Res 63: 7883-7890, 2003.

17. Oyajobi BO, Anderson DM, Traianedes K, Williams PJ, Yoneda T and Mundy GR: Therapeutic efficacy of a soluble receptor activator of nuclear factor kappaB-IgG Fc fusion protein in suppressing bone resorption and hypercalcemia in a model of humoral hypercalcemia of malignancy. Cancer Res 61: 2572-2578, 2001

18. Wang BL, Liang H, Zheng F, Li XX, Liu YB and Dai CL: Recombinant soluble receptor activator of nuclear factor-kappaB inhibits parathyroid hormone-induced osteoclastogenesis in vitro. Sheng Li Xue Bao 59: 169-174, 2007.

19. Tang P, Zhang L, Xu M, Zhang L and Wang Y: Inhibition of the osteoclast activity with the application of recombinant murine RANK protein. Artif Cells Blood Substit Immobil Biotechnol 38 169-177, 2010.

20. Hefley TJ and Stern PH: Isolation of osteoclasts from fetal rat long bones. Calcif Tissue Int 34: 480-487, 1982.

21. Anderson DM, Maraskovsky E, Billingsley WL, et al: A homologue of the TNF receptor and its ligand enhance T-cell growth and dendritic-cell function. Nature 390: 175-179, 1997.

22. Vitovski S, Phillips JS, Sayers J and Croucher PI: Investigating the interaction between osteoprotegerin and receptor activator of NF-kappaB or tumor necrosis factor-related apoptosis-inducing ligand: evidence for a pivotal role for osteoprotegerin in regulating two distinct pathways. J Biol Chem 282: 31601-31609, 2007.

23. Zhang S, Liu C, Huang P, et al: The affinity of human RANK binding to its ligand RANKL. Arch Biochem Biophys 487: 49-53, 2009.

24. French DL, Muir JM and Webber CE: The ovariectomized, mature rat model of postmenopausal osteoporosis: an assessment of the bone sparing effects of curcumin. Phytomedicine 15: 1069-1078, 2008

25. Seibel MJ: Biochemical markers of bone turnover: Part I: Biochemistry and variability. Clin Biochem Rev 26: 97-122, 2005.

26. Szulc P and Bouxsein ML: Overview of osteoporosis: Epidemiology and clinical management. Veterbral Fracture Initiative Resource Document PART I, 2011.

27. Kanis JA, Melton LJ III, Christiansen C, Johnston CC and Khaltaev N: The diagnosis of osteoporosis. J Bone Miner Res 9: 1137-1141, 1994. 